\title{
Sets of Periods for Expanding Maps on Flat Manifolds
}

\author{
By
}

\author{
Roberto Tauraso, Firenze, Italy
}

\begin{abstract}
It is proven that the sets of periods for expanding maps on $n$ dimensional flat manifolds are uniformly cofinite, i.e. there is a positive integer $m_{0}$, which depends only on $n$, such that for any integer $m \geq m_{0}$, for any $n$-dimensional flat manifold $\mathcal{M}$ and for any expanding map $F$ on $\mathcal{M}$, there exists a periodic point of $F$ whose least period is exactly $m$.
\end{abstract}

Expanding maps were first introduced in a differentiable setting by M. Shub in [12], and then studied by D. Ruelle in [11] who proposed a more general definition based on a simple metric property: they are open continuous maps which locally expand distances. In general, it is rather difficult to prove the existence of at least an expanding map on a metric space, but there is a class of connected compact manifolds where the set of expanding maps is always nonempty: flat manifolds. The term flat derives from the fact that flat manifolds are connected Riemannian compact manifolds whose Levi-Civita connection has curvature that identically vanishes (e.g. the $n$-torus, the Klein bottle...).

Due to the strong topological properties of expanding maps on flat manifolds, in this note, I am able to determine the uniform cofiniteness of their sets of periods. This work has been inspired by the paper [7] where B. Jiang and J. Llibre studied the sets of periods for generic continuous maps of the $n$-torus and obtained a similar result in the expanding case.

I wish to thank the referee for very helpful comments and suggestions.

\section{Preliminaries.}

Let $\mathcal{M}$ be a compact connected topological $n$-dimensional manifold.

Definition 1.1 An open continuous map $F: \mathcal{M} \rightarrow \mathcal{M}$ is expanding if there exist a metric $d$ compatible with the topology of $\mathcal{M}$ and constants $\epsilon_{0}>0$, $\lambda>1$ such that for $x, x^{\prime} \in \mathcal{M}$

$$
d\left(x, x^{\prime}\right) \leq \epsilon_{0} \quad \text { implies } \quad d\left(F(x), F\left(x^{\prime}\right)\right) \geq \lambda d\left(x, x^{\prime}\right) .
$$

We will denote by $\mathcal{E}(\mathcal{M})$ the set of all maps expanding on $\mathcal{M}$.

1991 Mathematics Subject Classification. Primary 58F15; Secondary 55M20, 57S30.

Key words and phrases. Expanding maps, periodic points, flat manifolds. 
We briefly summarize the properties of expanding maps which will be useful later (see [12] for more details). Let $F \in \mathcal{E}(\mathcal{M})$ then

(i) $F$ is a self-covering map, $N$-to- 1 with $N \geq 2$;

(ii) $F^{k} \in \mathcal{E}(\mathcal{M})$ for all $k \geq 1$;

(iii) the set of fixed points $\operatorname{Fix}_{\mathcal{M}}(F) \stackrel{\text { def }}{=}\{x \in \mathcal{M}: F(x)=x\}$ is non-empty and finite, and the set of periodic points $\bigcup_{k \geq 1} \operatorname{Fix}_{\mathcal{M}}\left(F^{k}\right)$ is countable and dense in $\mathcal{M}$;

(iv) the homomorphism $\widetilde{F}^{\sharp}$ induced by $F$ on the deck transformation group of the universal covering space of $\mathcal{M}$ is injective and characterizes the topological properties of $F$. This means that expanding maps which induce the same homomorphism are topologically conjugate: if $\Phi \in \mathcal{E}(\mathcal{M})$ and $\widetilde{F}^{\sharp}=\widetilde{\Phi}^{\sharp}$ then there exists a homeomorphism $\alpha_{0}$ of $\mathcal{M}$ such that

$$
F=\alpha_{0}^{-1} \circ \Phi \circ \alpha_{0}
$$

In this note we are interested in a particular class of manifolds: flat manifolds (see [4] as a general reference).

Definition 1.2 A cocompact, torsion free, discrete subgroup $\Gamma$ of $O(n) \bowtie \mathbb{R}^{n}$, the group of the affine isometries of $\mathbb{R}^{n}$, is called a Bieberbach group and $\mathcal{M}=\mathbb{R}^{n} / \Gamma$ is the flat manifold associated to $\Gamma$.

The following statements will allow us to find a better representation of a given flat manifold and of its expanding maps. Let $\Gamma$ be a Bieberbach group then

(v) ([2]) the holonomy group of $\Gamma$, i. e. $\Psi \stackrel{\text { def }}{=} \Gamma /\left(\Gamma \cap\left(\{\mathbb{I}\} \ltimes \mathbb{R}^{n}\right)\right)$, has finite order $|\Psi|$;

(vi) ([3]) there is an element $(B, b)$ of the affine group $\operatorname{Aff}\left(\mathbb{R}^{n}\right)$, which conjugates $\Gamma$ to a subgroup $\Gamma^{\prime} \subset \operatorname{Aff}\left(\mathbb{R}^{n}\right)$, called affine Bieberbach group, such that for any $\gamma \in \Gamma^{\prime}$ :

$$
\gamma=(U, u) \quad \text { with } \quad U \in \operatorname{GL}(n, \mathbb{Z}) \text { and }|\Psi| u \in \mathbb{Z}^{n} .
$$

Note that $|\operatorname{det}(U)|=1$. Moreover, $\Gamma^{\prime} \cap\left(\{\mathbb{I}\} \ltimes \mathbb{R}^{n}\right)=\{\mathbb{I}\} \ltimes \mathbb{Z}^{n}$ and the holonomy group becames $\Psi^{\prime}=\Gamma^{\prime} /\left(\{\mathbb{I}\} \ltimes \mathbb{Z}^{n}\right)$;

(vii) ([8]) if $\varphi: \Gamma^{\prime} \rightarrow \Gamma^{\prime}$ is an injective homomorphism of the affine Bieberbach group $\Gamma^{\prime}$, there exists $(A, a) \in \mathbb{Z}^{n \times n} \ltimes \mathbb{R}^{n} \subset \operatorname{Aff}\left(\mathbb{R}^{n}\right)$ such that, for all $\gamma=(U, u) \in \Gamma^{\prime}$ :

$$
\varphi(\gamma)=(A, a)^{\sharp}(\gamma)=(A, a) \gamma(A, a)^{-1}=\left(A U A^{-1}, A u+\left(\mathbb{I}-A U A^{-1}\right) a\right) .
$$

Let $\mathcal{M}=\mathbb{R}^{n} / \Gamma$ be a flat manifold and let $\mathcal{M}^{\prime}$ be the quotient space $\mathbb{R}^{n} / \Gamma^{\prime}$, where $\Gamma^{\prime}=(B, b) \Gamma(B, b)^{-1}$ is the affine Bieberbach group given by (vi). Then, $(B, b)$ induces a homeomorphism from $\mathcal{M}$ onto $\mathcal{M}^{\prime}$. For this reason, from now on, the flat manifold $\mathcal{M}$ will be considered as the quotient space of $\mathbb{R}^{n}$ by the affine Bieberbach group $\Gamma^{\prime}$ rather than the Bieberbach group $\Gamma$. 
Let $F$ be an expanding map of $\mathcal{M}$ then, by (iv), $F$ induces an injective homomorphism $\varphi$ on the deck transformation group $\Gamma^{\prime}$ of the universal covering $\mathbb{R}^{n}$. By (vii), there is an affine map $(A, a)$, that is a lifting to $\mathbb{R}^{n}$ of a map $\Phi_{(A, a)} \in \mathcal{E}(\mathcal{M})$, which induces on $\Gamma^{\prime}$ a homomorphism $\Phi_{(A, a)}^{\sharp}$ equal to $\varphi$. Therefore, again by (iv), $F$ and $\Phi_{(A, a)}$ are topologically conjugate and we will say that $\Phi_{(A, a)}$ is the endomorphism associated to $F$. Note that, by (1), the map $\Phi_{(A, a)}$ is expanding iff all the eigenvalues of the integer matrix $A$ are outside the closed unit disc in $\mathbb{C}$.

Now we are ready to establish a result, proved by D. Epstein and M. Shub in [5], which really motivates the study of expanding maps just on flat manifolds.

Theorem 1.3 If $\mathcal{M}$ is a flat manifold, then $\mathcal{E}(\mathcal{M})$ is not empty.

Proof. The flat manifold $\mathcal{M}$ can be represented as quotient space $\mathbb{R}^{n} / \Gamma^{\prime}$ where $\Gamma^{\prime}$ is an affine Bieberbach group. The affine map $\left(\left(\left|\Psi^{\prime}\right|+1\right) \mathbb{I}, 0\right)$ induces on $\Gamma^{\prime}$ a homomorphism $\varphi$ such that, for all $\gamma=(U, u) \in \Gamma^{\prime}$,

$$
\varphi(\gamma)=\left(\left(\left|\Psi^{\prime}\right|+1\right) \mathbb{I}, 0\right) \gamma\left(\left(\left|\Psi^{\prime}\right|+1\right) \mathbb{I}, 0\right)^{-1}=\left(U,\left(\left|\Psi^{\prime}\right|+1\right) u\right)=\left(\mathbb{I},\left|\Psi^{\prime}\right| u\right)(U, u) .
$$

By (vi), $\left|\Psi^{\prime}\right| u \in \mathbb{Z}^{n}$, hence $\varphi(\gamma) \in \Gamma^{\prime}$.

Therefore, the affine map $\left(\left(\left|\Psi^{\prime}\right|+1\right) \mathbb{I}, 0\right)$ is the lifting of the map $\Phi_{\left.\left(\left|\Psi^{\prime}\right|+1\right) \sigma I, 0\right)}$ which belongs to $\mathcal{E}(\mathcal{M})$ because $\left(\left|\Psi^{\prime}\right|+1\right) \geq 2$.

\section{Fixed points.}

Let $\mathcal{M}$ be a flat manifold and let $F \in \mathcal{E}(\mathcal{M})$. We know by (iii) that the number of fixed points of $F$ is finite. Now, we want to compute exactly the number $\mathcal{N}(F) \stackrel{\text { def }}{=} \operatorname{card}\left(\operatorname{Fix}_{\mathcal{M}}(F)\right)$. The following remarks and the next lemma will be of value for this purpose.

Since, by (vi), $\mathbb{I} \ltimes \mathbb{Z}^{n}$ is a subgroup of the affine Bieberbach group $\Gamma^{\prime}$, then $\mathcal{M}$ is always covered by the torus $\mathbf{T}^{n} \stackrel{\text { def }}{=} \mathbb{R}^{n} /\left(\mathbb{I} \ltimes \mathbb{Z}^{n}\right)$. When this covering is not trivial, i. e. when $\Psi^{\prime} \neq\{(\mathbb{I}, 0)\}$, the manifold is called an infra-torus. If $\Phi_{(A, a)}$ is the endomorphism associated to $F$ then the following commutative diagram holds

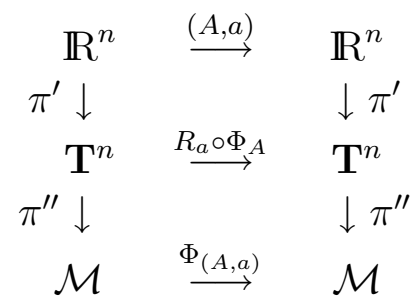

where: $R_{a}: \mathbf{T}^{n} \rightarrow \mathbf{T}^{n}$ is a toral rotation, $R_{a}(x)=x+a$ and $\Phi_{A}: \mathbf{T}^{n} \rightarrow \mathbf{T}^{n}$ is a toral linear endomorphism $\Phi_{A}(x)=A x$.

Lemma 2.1 Let $A$ be a matrix in $\mathbb{Z}^{n \times n}$ then: 
(a) if $A$ is non-singular then $\Phi_{A}$ is a self-covering of $\mathbf{T}^{n}$ with degree equal to $|\operatorname{det}(A)| \geq 1$;

(b) if the spectrum of $A$ has no roots of unity, $i$. e. $\operatorname{det}\left(A^{k}-\mathbb{I}\right) \neq 0$ for all $k \geq 1$ then

$$
\operatorname{card}\left(\operatorname{Fix}_{\mathbf{T}^{n}}\left(\Phi_{A}^{k}\right)\right)=\left|\operatorname{det}\left(A^{k}-\mathbb{I}\right)\right| \quad \forall k \geq 1 .
$$

Proof. (a) Since $A$ is non-singular, $\Phi_{A}$ is a self-covering of $\mathbf{T}^{n}$. Moreover there exist two matrices $P, Q \in \operatorname{GL}(n, \mathbb{Z})$ such that $A=P D Q$ where $D \in \mathbb{Z}^{n \times n}$ is diagonal (see [6] p. 384). This means that $\Phi_{A}=\Phi_{P} \circ \Phi_{D} \circ \Phi_{Q}$ where $\Phi_{P}$ and $\Phi_{Q}$ are homeomorphisms of $\mathbf{T}^{n}$. Hence

$$
\operatorname{card}\left(\Phi_{A}^{-1}(0)\right)=\operatorname{card}\left(\Phi_{D}^{-1}(0)\right)=|\operatorname{det}(D)|=|\operatorname{det}(A)| \geq 1 .
$$

(b) A point $x \in \operatorname{Fix}_{\mathbf{T}^{n}}\left(\Phi_{A}^{k}\right)$ iff there exists $y \in \mathbb{R}^{n}$ such that $\left(A^{k}-\mathbb{I}\right) y \in \mathbb{Z}^{n}$. Therefore, since $\operatorname{det}\left(A^{k}-\mathbb{I}\right) \neq 0$ for all $k \geq 1$,

$$
\operatorname{Fix}_{\mathbf{T}^{n}}\left(\Phi_{A}^{k}\right)=\operatorname{Ker}\left(\Phi_{\left(A^{k}-\sigma I\right)}\right)=\Phi_{\left(A^{k}-\sigma I\right)}^{-1}(0),
$$

and, by (a), $\operatorname{card}\left(\operatorname{Fix}_{\mathbf{T}^{n}}\left(\Phi_{A}^{k}\right)\right)=\left|\operatorname{det}\left(A^{k}-\mathbb{I}\right)\right|$.

Here is the theorem which gives the explicit formula for $\mathcal{N}(F)$.

Theorem 2.2 Let $\mathcal{M}$ be a flat manifold. If $F \in \mathcal{E}(\mathcal{M})$ and $\Phi_{(A, a)}$ is the endomorphism associated to $F$, then

$$
\mathcal{N}(F)=\frac{1}{\left|\Psi^{\prime}\right|} \sum_{U \in r\left(\Psi^{\prime}\right)}|\operatorname{det}(A-U)|
$$

where $r$ is the map that assigns to each $(U, u) \in \Psi^{\prime}$ its rotational part $U$.

Proof. Since the maps $F$ and $\Phi_{(A, a)}$ are topologically conjugate, $\mathcal{N}(F)=$ $\mathcal{N}\left(\Phi_{(A, a)}\right)$ and it is enough to compute the number of fixed points of $\Phi_{(A, a)}$. Since $x \in \operatorname{Fix}_{\mathcal{M}}\left(\Phi_{(A, a)}\right)$ iff there exist $y \in \mathbb{R}^{n}$ and $(U, u) \in \Gamma^{\prime}$ such that $(A, a)(y)=(U, u)(y)$ and $\pi^{\prime \prime}\left(\pi^{\prime}(y)\right)=x$,

$$
\operatorname{Fix}_{\mathcal{M}}\left(\Phi_{(A, a)}\right)=\pi^{\prime \prime} \circ \pi^{\prime}\left(\bigcup_{(U, u) \in \Gamma^{\prime}}(A-U)^{-1}(u-a)\right)=\pi^{\prime \prime}\left(\bigcup_{(U, u) \in \Psi^{\prime}} \Phi_{(A-U)}^{-1}\left(\pi^{\prime}(u-a)\right)\right) .
$$

Now, we show that if $(U, u)$ and $(V, v)$ are two different elements of $\Psi^{\prime}$ then

$$
\Phi_{(A-U)}^{-1}\left(\pi^{\prime}(u-a)\right) \cap \Phi_{(A-V)}^{-1}\left(\pi^{\prime}(v-a)\right)=\emptyset .
$$

Otherwise there exist $y \in \mathbb{R}^{n}$ and $p, q \in \mathbb{Z}^{n}$ such that

$$
\left\{\begin{array}{l}
(A-U) y=u-a+p \\
(A-V) y=v-a+q .
\end{array}\right.
$$


These equations yield $(V, v+q)^{-1}(U, u+p) y=y$. Since the action of $\Gamma^{\prime}$ on $\mathbb{R}^{n}$ is properly discontinuous, $(V, v+q)^{-1}(U, u+p)=(\mathbb{I}, 0)$ and $U=V$ contradicting the hypothesis. By $(3)$, since the degree of the covering $\pi^{\prime \prime}$ is equal to $\left|\Psi^{\prime}\right|$,

$$
\mathcal{N}\left(\Phi_{(A, a)}\right)=\frac{1}{\left|\Psi^{\prime}\right|} \sum_{U \in r\left(\Psi^{\prime}\right)} \operatorname{card}\left(\Phi_{(A-U)}^{-1}\left(\pi^{\prime}(u-a)\right)\right) .
$$

To complete the proof, it is enough to remark that $\operatorname{card}\left(\Phi_{(A-U)}^{-1}\left(\pi^{\prime}(u-a)\right)\right)=$ $|\operatorname{det}(A-U)|$ by the preceding lemma.

\section{Sets of periods and uniform cofiniteness.}

Definition 3.1 For $m \geq 1$, the number of periodic points of least period $m$ for $F$ is denoted by

$$
p_{F}(m) \stackrel{\text { def }}{=} \operatorname{card}\left(\operatorname{Fix}_{\mathcal{M}}\left(F^{m}\right) \backslash \bigcup_{k=1}^{m-1} \operatorname{Fix}_{\mathcal{M}}\left(F^{k}\right)\right) .
$$

The set of periods $\mathcal{P}(F)$ of the map $F$ is the set of positive integers $m$ such that $p_{F}(m)>0$.

By (iii), we know that $p_{F}(m)$ is finite for all $m \geq 1$ and $\mathcal{P}(F)$ is infinite. But some periods may be missing: for example, $\Phi_{-2 \sigma I} \in \mathcal{E}\left(\mathbf{T}^{n}\right)$ has no points of period 2:

$p_{\Phi_{-2 \mathrm{I}}}(2)=\mathcal{N}\left(\Phi_{-2 \sigma I}^{2}\right)-\mathcal{N}\left(\Phi_{-2 \sigma I}\right)=|\operatorname{det}(4 \mathbb{I}-\mathbb{I})|-|\operatorname{det}(-2 \mathbb{I}-\mathbb{I})|=3^{n}-3^{n}=0$.

However, B. Jiang and J. Llibre have proven in [7] that there is a positive integer $m_{0}$ such that for any integer $m \geq m_{0}$ and for any expanding map $F$ of $\mathbf{T}^{n}$ there exists a periodic point of $F$ whose least period is exactly $m$. In the next theorem we state that the above property is verified not only for $\mathbf{T}^{n}$ but for each $n$-dimensional flat manifold $\mathcal{M}$. The following lemma on algebraic numbers (see $[\mathbf{7}]$ and $[\mathbf{1 0}]$ ) is needed.

Lemma 3.2 Let $\alpha$ be a nonzero algebraic number with minimal polynomial $Q \in \mathbb{Z}[x]$ of degree $d$. If $|\alpha| \neq 1$ then

$$
|| \alpha|-1| \geq \frac{1}{2^{d^{2}} M(\alpha)^{d}}
$$

with $M(\alpha) \stackrel{\text { def }}{=}|a| \prod_{i=1}^{d} \max \left\{1,\left|\alpha_{i}\right|\right\}$ where $a$ is the leading coefficient of $Q$ and $\alpha_{1}, \ldots, \alpha_{d}$ the roots of $Q$.

Here is the main result of this note. 
Theorem 3.3 Let $n$ be a positive integer. Then the sets of periods for expanding maps on n-dimensional flat manifolds are uniformly cofinite, i.e. there is a positive integer $m_{0}$, which depends only on $n$, such that for any integer $m \geq m_{0}$, for any $n$-dimensional flat manifold $\mathcal{M}$ and for any expanding map $F$ on $\mathcal{M}$, there exists a periodic point of $F$ whose least period is exactly $m$.

Proof. Let $\mathcal{M}$ be a $n$-dimensional flat manifold and let $F \in \mathcal{E}(\mathcal{M})$ with $\Phi_{(A, a)}$ the associated endomorphism. Suppose that $\alpha_{1}, \ldots, \alpha_{n}$ are the eigenvalues of $A$ and let $\varrho(A) \stackrel{\text { def }}{=} \max \left\{\left|\alpha_{1}\right|, \ldots,\left|\alpha_{n}\right|\right\}$.

First observe that if $U \in r\left(\Psi^{\prime}\right)$ and $k \geq 1$ then

$$
\left(A^{k} U^{-1}\right)^{j}=\left(A^{k} U^{-1} A^{-k}\right)\left(A^{2 k} U^{-1} A^{-2 k}\right) \ldots\left(A^{j k} U^{-1} A^{-j k}\right) A^{j k} \quad \forall j \geq 1 .
$$

Since, by (v) and (vi), $A r\left(\Psi^{\prime}\right) A^{-1} \subset r\left(\Psi^{\prime}\right)$ and $r\left(\Psi^{\prime}\right)$ is a finite group of order $\left|\Psi^{\prime}\right|$, there is an integer $1 \leq j_{0} \leq\left|\Psi^{\prime}\right|$ such that $A^{j_{0} k} U^{-1} A^{-j_{0} k}=U^{-1}$. Let

$$
V=\left(A^{k} U^{-1} A^{-k}\right)\left(A^{2 k} U^{-1} A^{-2 k}\right) \ldots\left(A^{j_{0} k} U^{-1} A^{-j_{0} k}\right) .
$$

Then $V \in r\left(\Psi^{\prime}\right)$ and therefore $V^{\left|\Psi^{\prime}\right|}=\mathbb{I}$. Hence, by (4),

$$
\left(A^{k} U^{-1}\right)^{j_{0}\left|\Psi^{\prime}\right|}=V^{\left|\Psi^{\prime}\right|} A^{k j_{0}\left|\Psi^{\prime}\right|}=\left(A^{k}\right)^{j_{0}\left|\Psi^{\prime}\right|} .
$$

This means that, in absolute value, the eigenvalues of $A^{k} U^{-1}$ and $A^{k}$ are the same: $\left|\alpha_{1}\right|^{k}, \ldots,\left|\alpha_{n}\right|^{k}$.

Since, by (vi), $|\operatorname{det}(U)|=1$ for all $U \in r\left(\Psi^{\prime}\right)$, it follows from (2) that

$$
\mathcal{N}\left(F^{k}\right)=\frac{1}{\left|\Psi^{\prime}\right|} \sum_{U \in r\left(\Psi^{\prime}\right)}\left|\operatorname{det}\left(A^{k}-U\right)\right|=\frac{1}{\left|\Psi^{\prime}\right|} \sum_{U \in r\left(\Psi^{\prime}\right)}\left|\operatorname{det}\left(A^{k} U^{-1}-\mathbb{I}\right)\right|
$$

Therefore, for $m, k \geq 1$

$$
\frac{\mathcal{N}\left(F^{m}\right)}{\mathcal{N}\left(F^{k}\right)}=\frac{\sum_{U \in r\left(\Psi^{\prime}\right)}\left|\operatorname{det}\left(A^{m} U^{-1}-\mathbb{I}\right)\right|}{\sum_{U \in r\left(\Psi^{\prime}\right)}\left|\operatorname{det}\left(A^{k} U^{-1}-\mathbb{I}\right)\right|} \geq \prod_{i=1}^{n} \frac{\left|\alpha_{i}\right|^{m}-1}{\left|\alpha_{i}\right|^{k}+1} .
$$

The eigenvalues $\alpha_{1}, \ldots, \alpha_{n}$ are algebraic numbers greater than 1 in absolute value: the minimal polynomial of each $\alpha_{i}$ is monic, has degree $d_{i} \leq n$ and therefore $2 \leq M\left(\alpha_{i}\right) \leq \varrho(A)^{n}$. Hence, by the previous lemma,

$$
\left|\alpha_{i}\right|-1 \geq \frac{1}{2^{d_{i}^{2}} M\left(\alpha_{i}\right)^{d_{i}}} \geq \frac{1}{2^{n^{2}} \varrho(A)^{n^{2}}} .
$$

Let $1 \leq k \leq \frac{m}{2}$. Then

$$
\frac{\left|\alpha_{i}\right|^{m}-1}{\left|\alpha_{i}\right|^{k}+1} \geq\left|\alpha_{i}\right|^{k} \frac{\left|\alpha_{i}\right|^{m-k}-1}{\left|\alpha_{i}\right|^{k}+1} \geq\left|\alpha_{i}\right|^{k} \frac{\left|\alpha_{i}\right|-1}{\left|\alpha_{i}\right|^{k}+1} \geq \frac{\left|\alpha_{i}\right|-1}{2} \geq \frac{1}{2^{n^{2}+1} \varrho(A)^{n^{2}}},
$$


and it follows from (5) that

$$
\frac{\mathcal{N}\left(F^{m}\right)}{\mathcal{N}\left(F^{k}\right)} \geq\left(\frac{1}{2^{n^{2}+1} \varrho(A)^{n^{2}}}\right)^{n-1} \frac{\varrho(A)^{m}-1}{\varrho(A)^{k}+1} \geq \frac{\varrho(A)^{\frac{m}{2}}-1}{2^{n^{3}} \varrho(A)^{n^{3}}} .
$$

The right member of the above inequality is an increasing function with respect to $\varrho(A)$ for $m \geq 2 n^{3}$. Thus there is $m_{0} \geq 2 n^{3}$, which depends only on the dimension $n$, such that the inequality

$$
\frac{\mathcal{N}\left(F^{m}\right)}{\mathcal{N}\left(F^{k}\right)} \geq \frac{\varrho(A)^{\frac{m}{2}}-1}{2^{n^{3}} \varrho(A)^{n^{3}}} \geq \frac{2^{\frac{m}{2 n}}-1}{2^{n^{3}+n^{2}}}>\frac{m}{2}
$$

holds for all $m \geq m_{0}$.

Let $x \in \mathcal{M}$ be a fixed point of $F^{m}$. Then it has a least period $k$ with $1 \leq k \leq m$. Moreover $k$ divides $m$ : indeed $m=q k+r$ with $q \geq 0$ and $0 \leq r<k$, so $x=F^{m}(x)=F^{r}\left(F^{q k}(x)\right)=F^{r}(x)$, which implies that $r=0$ by the minimality of $k$. Therefore

$$
p_{F}(m)=\operatorname{card}\left(\operatorname{Fix}_{\mathcal{M}}\left(F^{m}\right) \backslash \bigcup_{k \mid m, k<m} \operatorname{Fix}_{\mathcal{M}}\left(F^{k}\right)\right),
$$

and, since the conditions $k \mid m$ and $k<m$ imply that $1 \leq k \leq \frac{m}{2}$, we obtain by inequality (6)

$$
p_{F}(m) \geq \mathcal{N}\left(F^{m}\right)-\sum_{k \mid m, k<m} \mathcal{N}\left(F^{k}\right)>\mathcal{N}\left(F^{m}\right)\left(1-\sum_{1 \leq k \leq \frac{m}{2}} \frac{2}{m}\right)=0,
$$

that is $m \in \mathcal{P}(F)$ for $m \geq m_{0}$.

As a final remark, we give the complete list of all the missing periods for expanding maps on flat manifolds up to dimension 3 (for higher dimensions there are no results). As regards the $n$-torus, the situation is summarized in the following table (see $[\mathbf{1}]$ and $[\mathbf{7}]$ ).

\begin{tabular}{|c|c|c|}
\hline Torus & Characteristic Polynomial of $A$ & $\mathbb{N}^{*} \backslash \mathcal{P}\left(\Phi_{A}\right)$ \\
\hline $\mathbf{T}^{1}$ & $x+2$ & 2 \\
\hline $\mathbf{T}^{2}$ & $x^{2}+2 x+2$ & 2,3 \\
\cline { 2 - 3 } & $x^{2}+2$ & 4 \\
\hline \multirow{3}{*}{$\mathbf{T}^{3}$} & $x^{3}+2$ & 2,6 \\
\cline { 2 - 3 } & $x^{3}-2$ & 3 \\
\cline { 2 - 3 } & $x^{3}+x^{2}+x+2$ & 2,4 \\
\cline { 2 - 3 } & $x^{3}+x^{2}+2$ & 5 \\
\hline
\end{tabular}

On the other hand, if we consider an $n$-infra-torus $\mathcal{M}$ then $\mathcal{P}(F)=\mathbb{N}^{*}$ for all $F \in \mathcal{E}(\mathcal{M})$ and $n \leq 3$ (see $[\mathbf{9}]$ for $n=2$ and [13] for $n=3$ ). 


\section{References}

[1] Alsedà L, Baldwin S, Llibre J, Swanson R, Szlenk W (1993) Torus maps and Nielsen numbers. Contemporary Math 152: 1-7

[2] Auslander L (1960) Bieberbach theorems on space groups and discrete uniform subgroups of Lie groups. Ann Math 71: 579-590

[3] Brown H, Bülow R, Neubüser J, Wondratschek H, Zassenhaus H (1978) Crystallographic groups of four dimensional space. New York: Wiley

[4] Charlap LS (1986) Bieberbach Groups and Flat Manifolds. New York: Springer-Verlag

[5] Epstein D, Shub M (1968) Expanding endomorphisms of flat manifolds. Topology 7: 139-141

[6] HuA LK (1982) Introduction to Number Theory. Berlin: Springer-Verlag

[7] Jiang B, Llibre J (1998) Minimal sets of periods for torus maps. Discrete and Continuous Dyn. Sys. 4: 301-320

[8] Lee KB, Shin J, Yokura S (1993) Free actions of finite abelian groups on the 3-torus. Topology Appl 53: 153-175

[9] Llibre J (1993) A note on the set of periods for Klein bottle maps. Pacific J Math 157: 87-93

[10] Mignotte M, Waldschmidt M (1993) On algebraic numbers of small height: linear forms in one logarithm. J Number Theory 47: 43-62

[11] Ruelle D (1978) The thermodynamic formalism. Reading: AddisonWesley

[12] Shub M (1969) Endomorphisms of compact differentiable manifolds. Amer J Math 91: 175-199

[13] Tauraso R (1996), Periodic points for expanding maps and for their extensions. Pisa: Ph.D. thesis, Scuola Normale Superiore

R. TAuraso

Dipartimento di Matematica "U. Dini"

Viale Morgagni, 67/A

50134 Firenze

Italy

e-mail:

tauraso@sns.it

tauraso@udini.math.unifi.it 